\title{
The Trend of Direct Medical Cost of Meliodiosis Patients in Kedah: A Retrospective Study from 2014 to 2017
}

This article was published in the following Dove Press journal: ClinicoEconomics and Outcomes Research

\author{
Kamaruddin Mardhiah (D) ${ }^{1,2}$ \\ Nadiah Wan-Arfah ${ }^{2}$ \\ Nyi Nyi Naing ${ }^{3}$ \\ Muhammad Radzi Abu Hassan ${ }^{4}$ \\ Huan-Keat Chan ${ }^{4}$ \\ Hazriah Hasan' \\ 'Faculty of Entrepreneurship and \\ Business, Universiti Malaysia Kelantan, \\ Kota Bharu, Kelantan, Malaysia; ${ }^{2}$ Faculty \\ of Health Sciences, Universiti Sultan \\ Zainal Abidin, Kuala Terengganu, \\ Terengganu, Malaysia; ${ }^{3}$ Faculty of \\ Medicine, Universiti Sultan Zainal Abidin, \\ Kuala Terengganu, Terengganu, Malaysia; \\ ${ }^{4}$ Clinical Research Center, Hospital \\ Sultanah Bahiyah, Ministry of Health \\ Malaysia, Alor Setar, Kedah, Malaysia
}

Purpose: This study was conducted to determine the direct medical cost of treating melioidosis patients. The calculation was made according to the variables extracted from medical records.

Materials and Methods: Data collection was performed retrospectively on a total of 293 cases from Hospital Sultanah Bahiyah, Kedah, Malaysia. The data consisted of personal information, treatment history, and investigation findings, including blood results, USG abdomen results, and CT scan results. The site of culture and sensitivity were also obtained. The total direct medical cost was based on the antibiotics/treatments received by the patients, diagnostic test and investigations performed. The trend analysis used to see the pattern of costs from 2014 to 2017. All the costs were compared based on patients' status and duration of stay at the hospital using the independent $t$-test.

Results: The overall mean of direct medical cost for melioidosis amounted to US \$233.61 (RM931.33). Overall, the finding confirms a huge reduction (44.7\%) of direct medical cost from 2014 to $2017(P=0.001)$. From 2015 to 2016, there was a 19.1\% reduction of direct medical cost $(P>0.95)$, followed by a 38.8\% reduction in costs from 2016 to 2017 ( $P=$ $0.019)$. In the case of the duration of stay, the mean of total direct medical cost among patients with $\geq 14$ duration of stay was higher compared to those with $<14$ duration of stay $(p<0.001)$. There was no significant mean difference of direct medical cost between patients who were cured and died.

Conclusion: Despite the higher mortality of melioidosis cases compared to other infectious diseases, there is a limitation in the amount of published data on the management cost of melioidosis. The importance of cost in managing this disease should be underlined to perform a fully prepared management toward the disease.

Keywords: melioidosis, direct medical cost, trend analysis

\section{Introduction}

Melioidosis affects the population in the tropical areas, mainly the countries positioned at the $20^{\circ} \mathrm{N}$ and $20^{\circ} \mathrm{S}$ latitudes, Southeast Asia, Southern China, Hong Kong, Taiwan, Northern Australia, and Papua New Guinea, and India. ${ }^{1}$ This disease is caused by a type of bacteria known as Burkholderia pseudomallei. Despite the increase in the number of infected individuals, the disease remained an underrecognised health problem. ${ }^{2}$ The incidence of melioidosis is also increasing in the Southeast Asian countries, especially Malaysia, Thailand, Vietnam, and Singapore, ${ }^{3}$ with approximately $40 \%$ cases reported in the endemic area. ${ }^{4}$ However, it was also
Correspondence: Nadiah Wan-Arfah Faculty of Health Sciences, Universiti Sultan Zainal Abidin, Gong Badak Campus, Kuala Nerus, Kuala Terengganu, Terengganu, 21300, Malaysia

Tel +60I2-6260508

Email wanwaj@unisza.edu.my 
reported that the number of cases and deaths of melioidosis was higher during the wet season in Malaysia. ${ }^{5}$ It was also endemic in areas with contaminated soil and water due to the presence of $B$. pseudomallei in these areas. ${ }^{4}$ Moreover, melioidosis is common in Northern Australia, Africa, India, and China. ${ }^{1}$ Based on the published case report in Malaysia from 1975 to 2015, it was reported that from 29 cases were died, which contributed to an increase in mortality rate to $43 \% .{ }^{6}$ In the 1980 s in Malaysia, this disease, particularly the septicaemic form of it, was associated with a high mortality rate $(65 \%)$ in the hospital. However, this rate was reduced to $19-37 \%$ due to the new treatment being implemented. ${ }^{6}$

The mortality rate of melioidosis in endemic countries remains high, which results in increased economic burden and productivity loss. ${ }^{7}$ As a solution, thorough treatment and management in the acute stage of this disease are essential, including a continuous eradication treatment. ${ }^{8}$ Hospital costs and economic burden have become critical issues. ${ }^{9}$ Based on the previous retrospective studies, 28 of melioidosis patients $(32.9 \%)$ required an intensive care unit (ICU) admission at a teaching hospital in Kuala Lumpur from 1988 to $2010,{ }^{10}$ while 27 patients (20.3\%) had to be admitted to the ICU at Singapore General Hospital from 1996 to $2002 .{ }^{11}$ Consequently, admission to ICU increased the overall cost of treatment. ${ }^{8}$ Furthermore, a limited number of studies in Malaysia on melioidosis were published, and no published journal has reported on the cost of treating the melioidosis or the economic burden of melioidosis. Notably, medical cost is also related to the duration of stay at the hospital, ${ }^{12}$ which is an essential parameter for measuring the source utilised to predict future costs. ${ }^{12}$ Accordingly, this study aims to describe the direct medical cost of melioidosis treatment per patient according to the year 2014 to 2017 and compare the costs with mortality and duration of stay using the data collected at Hospital Sultanah Bahiyah, Kedah, Malaysia.

\section{Materials and Methods}

\section{Ethics Approval}

An ethics approval for this research was obtained from Universiti Zainal Abidin Human Research Ethics Committee (UHREC) (UniSZA/UHREC/2019/119; dated October 21, 2019) and Medical Research and Ethics Committee (MREC), Ministry of Health Malaysia (MOH) (NMRR-19-3090-46158 (IIR); dated: November 29, 2019).
For the ethics approval, the consent form was not mandatory for the secondary data. The data was collected based on reviewing the Melioidosis Registry; the researcher did not have to access the patients' personal information. All procedures performed in this study were in accordance with the ethical standards of the institution and Malaysian research committee and with the 1964 Helsinki declaration.

\section{Data Collection}

A retrospective cohort research was performed on 293 inpatients in Hospital Sultanah Bahiyah, Kedah, Malaysia, from 2014 to 2017. Direct medical cost per patient was estimated from Melioidosis Registry from 2014 to 2017 and presented as rounded to US Dolar. All the calculated costs were based on the routine procedure applied for the melioidosis patient once admitted and diagnosed with melioidosis. Any costs resulting from the illness but were not directly involved with purchasing the medical services were considered as direct non-medical costs. The direct non-medical cost includes travel costs, meals, and accommodation were not calculated in the study. This research identified the trend of direct medical cost from 2014 until 2017. The direct medical cost of managing melioidosis for the patients who were survived or died based on length of stay was also compared. The patients selected in this study were 15 years old and older, and all types of bacteremia or non-bacteremia melioidosis were considered in the data collection. However, patients with HIV and died upon admission at the hospital were excluded. The medications received other than to treat melioidosis were not included in the study.

The patients were specified into two categories, namely; 1) patients who survived from the disease and were discharged from the hospital and 2) patients who died from the illness, and the date of death was verified by the medical doctors. The duration of stay was determined through the subtraction of the admission date and the date of patients' release from the hospital. This duration was specified into two, namely; 1) shorter than 14 days and 2) equal to 14 days and longer. The use of these categories was based on basic practice. ${ }^{12}$ Following that, the total direct medical cost was generally calculated by the antibiotic/treatment received, investigation, and diagnostic test applied to the patient during the admission to the hospital. The antibiotics prescription was recorded to identify the overall cost of the treatment received using the latest costing applied for inpatients in the selected hospital. The latest costing for this hospital represents all the 
government hospitals in Malaysia. The investigation cost consisted of the laboratory test (including FBC, UFEME, renal and liver functions, and blood sugar), CT scan, USG abdomen, and X-ray. For the purpose of the registry and research, it is required that an abdominal CT scan be done for every melioidosis patient in Malaysia to diagnose prostatic abscess. ${ }^{13}$ The diagnostic cost comprised the site of culture and sensitivity performed towards the drug. Specifically, the diagnosis was determined by culturepositive samples, divided into several sites, including blood, tissue, urine, pus/wound, sputum, stool, and bone. The patients were categorized into bacteremic and nonbacteremic based on the site of culture-positive performed. Bacteremic melioidosis was defined as patients with blood culture positive for $B$. pseudomallei. In contrast, nonbacteremic melioidosis was patients with a positive B. pseudomallei when organism was isolated from other than blood culture. All costs in 2014-2017 were adjusted for inflation by using the Consumer Price Index (CPI) for healthcare in Malaysia. All costs were standardized to year 2017 Malaysian Ringgit (RM) and the equivalent costs in 2017 US dollars were obtained using a conversion rate of US \$1 = RM4.1649.

\section{Statistical Analysis}

For data organisation and analysis, IBM SPSS Statistics (26.0) was used, while the categorical variables were described by the frequency (percentage) and numerical variables through the mean and standard deviation. Normality and equal variance assumptions were tested, which were followed by an independent $t$-test used to identify the mean difference of cost between the patients' status, duration of stay, and type of melioidosis. The logarithm transformation was applied to the skewed data and back transformation using delta method applied to interpret the final results. ${ }^{14}$ The Pearson chi-square was used to compare the proportion of survival status between the years. In the case of the trend analysis, the data were expressed as numerical variables according to the year. A sequence chart analysis was conducted to see the pattern of the direct medical cost from 2014 to 2017 based on the month. This was followed by the use of trend analysis to identify the reduction or increment of the direct medical cost from 2014 to 2017. The mean difference of direct medical cost based on year was determined by using repeated measure ANOVA. The power of the study was set as $80 \%$, and the level of significance was set at 0.05 . The Cohen's effect size calculated for the direct medical cost was 0.2 and 0.5 for the patient's status and duration of stay, respectively.

\section{Results \\ Descriptive Analysis}

A total of 293 melioidosis patients who fulfilled the inclusion and exclusion criteria were selected in the study. As shown in Table 1, 233 of the selected patients (79.5\%) were male, while 247 of the patients were Malay (84.3\%), which was the majority. While the mean (SD) age of the patients was 50.25 (16.2) years old, the range of age for the majority of the patients was from 15 to 64 years old. However, a total of 146 patients $(49.8 \%)$ died from melioidosis. Most of the patients $(87.4 \%)$ were diagnosed with blood culture, while the other 27 patients $(9.2 \%)$ and 22 patients $(7.5 \%)$ where the culture was done at pus/wound and tissue site, respectively. A laboratory test was required for all patients; $38.6 \%, 47.1 \%$, and $23.2 \%$ of patients underwent the ultrasound, X-ray, and CT scan, respectively. The survival status between years statistically has no difference (Table 2).

\section{The Trend of Direct Medical Cost from 2014 to 2017}

The trend of average direct medical cost for each month from 2014 to 2017 is shown in Figure 1, with the highest costs reported in October 2014, January 2015, and March 2016. Meanwhile, the trend of direct medical cost based on year is illustrated in Figure 2. The trend analysis

Table I Characteristics of the Patients with Melioidosis from 2010 to 2017 at Hospital Sultanah Bahiyah, Kedah $(n=293)$

\begin{tabular}{|c|c|}
\hline Demographic & n (\%) \\
\hline \multicolumn{2}{|l|}{ Gender } \\
\hline Male & $233(79.5)$ \\
\hline Female & $60(20.5)$ \\
\hline \multicolumn{2}{|l|}{ Race } \\
\hline Malay & $247(84.3)$ \\
\hline Chinese & $12(4.1)$ \\
\hline Indian & $27(9.2)$ \\
\hline Others & $7(2.4)$ \\
\hline Mean age in years $(S D)$ & $50.25(16.2)$ \\
\hline \multicolumn{2}{|l|}{ Age group (years) } \\
\hline $15-64$ & $24 \mid(82.3)$ \\
\hline$\geq 65$ & $52(17.7)$ \\
\hline Fatal outcome & I 46 (49.8) \\
\hline
\end{tabular}


Table 2 Comparison of Survival Status in Melioidosis Patients from 2010 to 2017 at Hospital Sultanah Bahiyah, Kedah $(n=293)$

\begin{tabular}{|l|l|l|l|}
\hline Year & Cured (n=147) n(\%) & Died (n= 1 46) n(\%) & p-value $^{\mathbf{a}}$ \\
\hline 2014 & $30(20.4)$ & $40(27.4)$ & 0.582 \\
2015 & $52(35.4)$ & $47(32.2)$ & \\
2016 & $38(25.9)$ & $35(24.0)$ & \\
2017 & $27(18.4)$ & $24(16.4)$ & \\
\hline
\end{tabular}

Note: ${ }^{\text {aPearson }}$ chi-square applied.

(Figure 2) revealed a $44.7 \%$ reduction of direct medical cost from 2014 to 2017 ( $P=0.001$ ). From 2015 to 2016, there was a $19.1 \%$ reduction of direct medical cost $(P>0.95)$, followed by a $38.8 \%$ reduction of costs from 2016 to $2017(P=0.019)$. There was an increment of direct medical cost from 2014 to 2015 , with $11.8 \%$ $(P>0.95)$.

\section{The Comparison of Direct Medical Cost Between Groups}

Although the decline of costs took place from 2014 to 2017, there was no significant mean difference in the total direct medical cost between the patients who were survived and those who died from the disease $(p=0.158)$. However, patients who survived exhibited a significantly higher mean of diagnostic costs than the patients who died (see Table 3). In comparing the costs between the duration of stay, antibiotic provision, and investigation/procedure, the significant overall cost was achieved (Table 4). Therefore, it could be concluded that a longer duration of stay leads to higher treatment costs. The diagnostic costs were significantly different between non-bacteremic and bacteremic patients (Table 5). The non-bacteremic patients show a higher mean of diagnostic costs compared to bacteremic patients.

\section{Discussion}

Thorough antimicrobial treatment in the critical stage of melioidosis and a long-term eradication treatment is essential. $^{2}$ The increase in melioidosis cases in the endemic areas in Malaysia possibly contributes to massive economic difficulties. Following the high number of hospitalised patients and mortality rates due to this disease, expensive treatment and cost are needed, which may result in long-term economic effects on the society, including the loss of productivity. ${ }^{8}$

It was recorded in this study that the highest average direct medical cost increased from US \$742.88 (RM3094) in March 2016, US \$700.14 (RM2916) in October 2014, and US \$1243.73 (RM5180) in January 2015. The results have shown a similar outcome reported by Hassan et al (2010). The increased number of the cases during these

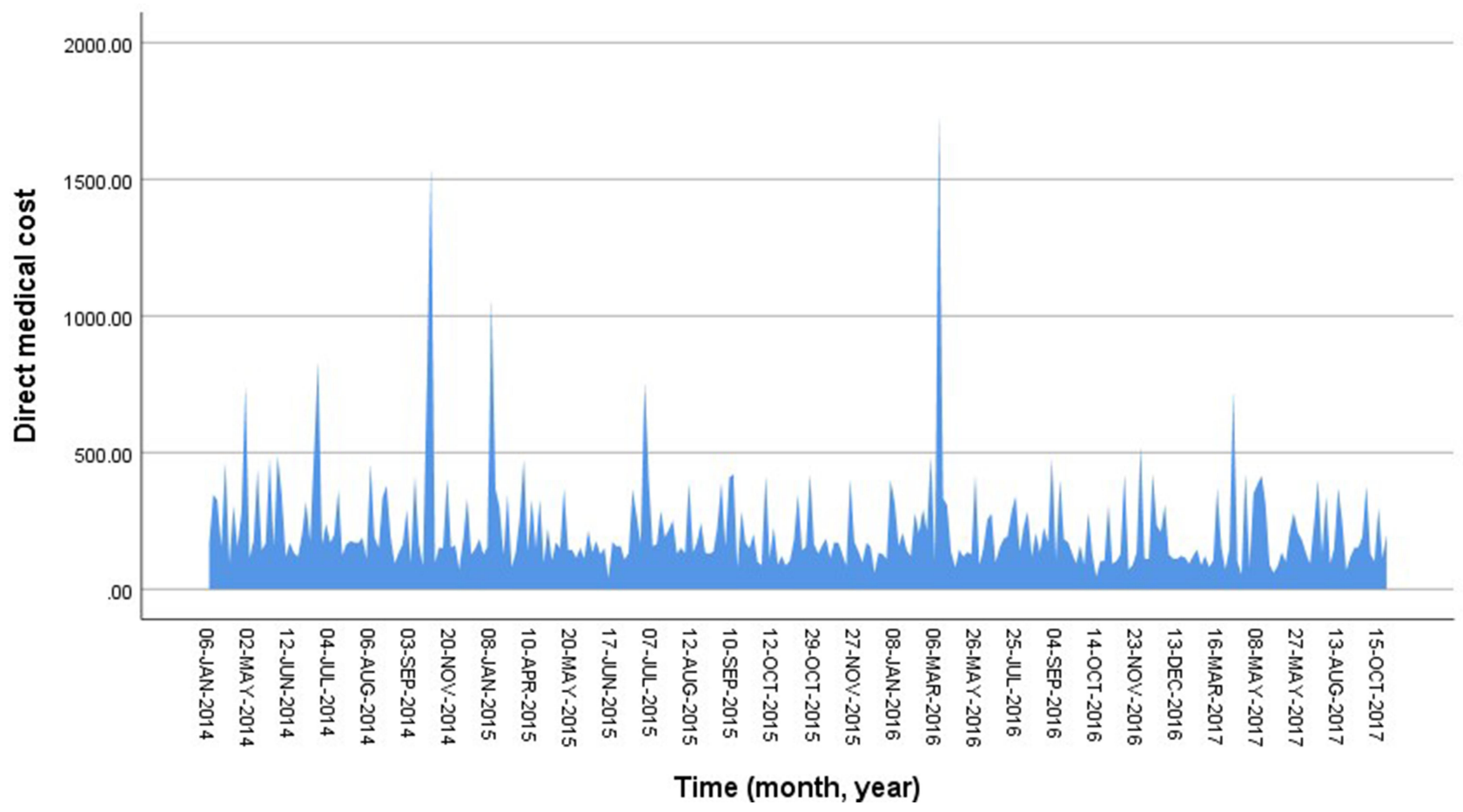

Figure I The trend of the average direct medical cost (USD) from 2014 to 2017 based on months. 


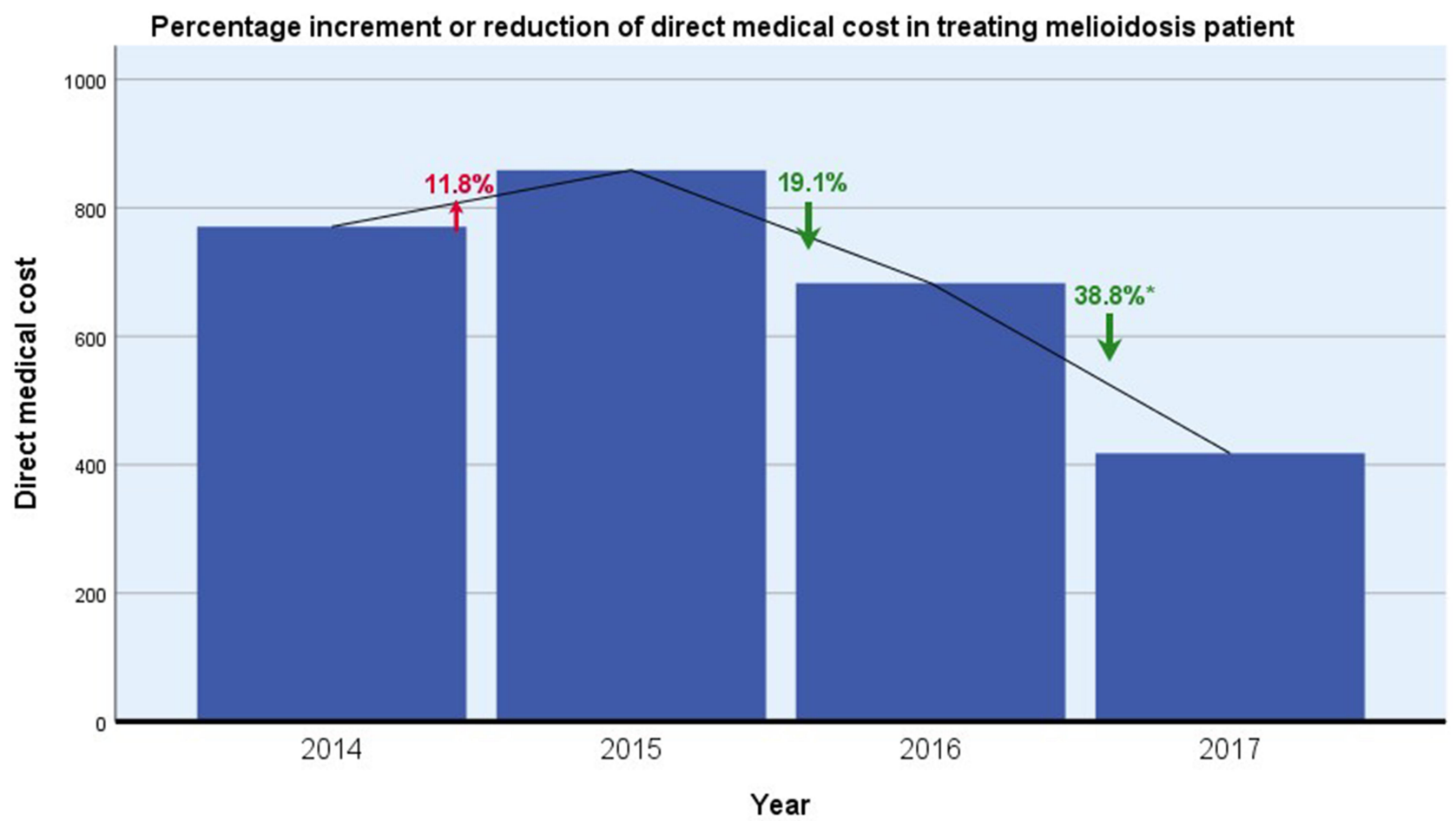

${ }^{*} p$-value $<0.05$

Figure 2 The percent of increment/reduction of direct medical cost.

months can be related to the occurrence of high rainfall. ${ }^{5}$ Based on that study, it was reported that the mean monthly rainfall was associated with the increase of melioidosis cases. ${ }^{5}$ A study conducted in Hospital Universiti Sains Malaysia, Kubang Kerian, Malaysia, also reported almost the same results where the highest admission of melioidosis patients was observed during November, December, January, and February. ${ }^{15}$ The rainy season in Malaysia usually started from November till February, which the risk of flooding is high. Published studies proposed that these wet season may enable the spreading of

Table 3 The Comparison of Costs Based on the Patients' Survival (Cured or Died from Disease) $(n=293)$

\begin{tabular}{|l|l|l|l|}
\hline Variable & $\begin{array}{c}\text { The Mean(SE) } \\
\text { of Died } \\
\text { Patients n= 146 }\end{array}$ & $\begin{array}{c}\text { The Mean(SE) } \\
\text { of Cured } \\
\text { Patients n=147 }\end{array}$ & p-value $^{\mathbf{a}}$ \\
\hline Antibiotic cost & $43.73(0.63)$ & $48.13(0.84)$ & 0.303 \\
Diagnostic cost & $9.71(0.09)$ & $11.33(0.08)$ & 0.003 \\
Investigation cost & $156.98(0.54)$ & $177.25(0.39)$ & 0.131 \\
Direct medical cost & $210.42(0.64)$ & $236.71(0.69)$ & 0.158 \\
\hline
\end{tabular}

Notes: andependent $t$-test applied; the logarithmic transformation was applied for the skewed data. Means and SEs were obtained by back-transformation via the delta method. The costs estimated per patient was presented in US Dollars.
B. pesudomallei in the environment and increased the chance of getting the infection from this bacteria. ${ }^{16,17}$

Despite the fact that we cannot identify the total economic burden of melioidosis patients in Malaysia since the calculation of the costs was only performed based on the direct medical cost, yet the average of these costs obtained for the study was considered higher for each patient. It was also found that direct medical cost constituted $14-25 \%$ of the overall economic difficulties from melioidosis. ${ }^{8}$ Based on research performed in Thailand, the average annual direct medical cost were associated with the cases of

Table 4 The Comparison of Costs Based on the Duration of Stay $(n=293)$

\begin{tabular}{|l|l|l|l|}
\hline Variable & $\begin{array}{c}\text { 0-I3 Days } \\
\text { Mean (SE) } \\
\mathbf{n = 1 4 9}\end{array}$ & $\begin{array}{c}\geq \text { I4 Days } \\
\text { Mean (SE) } \\
\mathbf{n = 1 4 4}\end{array}$ & p-value $^{\mathbf{a}}$ \\
\hline Antibiotic cost & $12.78(0.64)$ & $80.26(0.54)$ & $<0.00 \mathrm{I}$ \\
Diagnostic cost & $10.37(0.08)$ & $10.69(0.08)$ & 0.938 \\
Investigation cost & $156.01(0.45)$ & $178.67(0.48)$ & 0.312 \\
Direct medical cost & $179.16(0.53)$ & $269.61(0.65)$ & $<0.001$ \\
\hline
\end{tabular}

Notes: ${ }^{a}$ Independent $t$-test applied; the logarithmic transformation was applied for the skewed data. Means and SEs were obtained by back-transformation via the delta method. The costs estimated per patient was presented in US Dollars. 
Table 5 The Comparison of Costs Based on the Type of Melioidosis Patients in Survival Group $(n=147)$

\begin{tabular}{|l|l|l|l|}
\hline Variable & $\begin{array}{c}\text { Non- } \\
\text { Bacteremic } \\
\text { Mean (SE) } \\
\mathbf{n = 2 6}\end{array}$ & $\begin{array}{c}\text { Bacteremic } \\
\text { Mean (SE) } \\
\mathbf{n = 1 2 ~ I ~}\end{array}$ & p-value \\
\hline Antibiotic cost & $33.35(4.23)$ & $51.31(0.57)$ & $>0.95$ \\
Diagnostic cost & $18.46(0.13)$ & $9.80(0.06)$ & $<0.001$ \\
Investigation cost & $191.39(0.05)$ & $174.21(0.03)$ & 0.165 \\
Direct medical cost & $243.20(0.05)$ & $235.32(0.03)$ & 0.387 \\
\hline
\end{tabular}

Notes: ${ }^{a}$ Independent $t$-test applied; the logarithmic transformation was applied for the skewed data. Means and SEs were obtained by back-transformation via the delta method. The costs estimated per patient was presented in US Dollars.

hospitalised bacteremic melioidosis in $\mathrm{Sa}$ Kaeo and Nakhon Phanom. In Sa Kaeo, the treatment amounted to $\$ 37066$ (\$16,187 severe cases and \$20,876 non-severe cases), while $\$ 66,993$ cases $(\$ 17,178$ severe cases and $\$ 47,475$ non-severe cases) were reported in Nakhon. ${ }^{8}$

Notably, a higher value of sub costs was found among the patients who were survived from the disease compared to the individuals who died from the disease. This difference was possibly related to a longer duration of stay among the patients who were survived, as emphasised by Bhengsri et al (2013) that the average direct medical cost among nonfatal cases of melioidosis was higher compared to the cost among fatal cases in Thailand. Meanwhile, the lower costs in dead patients were possibly related to shorter duration of stay at the hospital, including the death of most melioidosis patients within 24 hours or 72 hours of admission, ${ }^{18,19}$ which was also not prepared for other clinical investigations or procedures. Moreover, a higher total direct medical cost were incurred to the patients with a duration of stay of longer or equal to 14 days at the hospital, in comparison to the patients with a duration of stay of shorter than 14 days. Overall, these results were possibly correlated to the difference in costs between the patients who were survived and those who died from the disease. It can be concluded that the longer the hospital stay, the more additional procedures and management to treat the melioidosis patient that lead to the increased costs.

The average antibiotic and diagnostic costs for this study among melioidosis patients amounted to US \$45.94 (RM191.34) and US \$10.52 (RM43.83), respectively. In the Malaysian context, the empirical antimicrobial treatment used to treat life-threatening melioidosis is meropenem. ${ }^{13,15}$ A study that was conducted in Thailand by Hantrakun et al (2015) reported that meropenem is more cost-effective in reducing the mortality rate of melioidosis by $9 \%$. In this study, meropenem and ceftazidime were compared to estimate the cost-effectiveness in treating acute severe melioidosis patients. ${ }^{20}$ It was another study in Vietnam emphasised on the cost-effectiveness of the use of selective media for the diagnosis of melioidosis. ${ }^{21}$ It was reported that approximately $\$ 100$ was incurred in Vientiane, while \$39 was incurred for each patient in Siem Reap for detecting melioidosis by using the selective culture. ${ }^{21}$ However, there was no published study on cost analysis or the cost-effectiveness of treating melioidosis in Malaysia. Based on this study, the mean diagnostic cost for bacteremic patients was statistically different from non-bacteremic patients among the survival group. The diagnostic cost was lower in bacteremic patients than non-bacteremic patients due to the diagnosis of this group mostly based on blood culture results only. For nonbacteremic patients, the sample was done for multiple culture sites that lead to a higher cost of diagnosis.

The trend analysis was used to estimate the direct medical cost for each year. It was indicated from the results that the direct medical cost for melioidosis patients were frequently reduced from 2014 until 2017. This phenomenon implied improved management for the diagnostic and treatment therapies. It could be seen that the forecast of the direct medical cost could be applied for future research as a reference to achieve better planning and use of adequate resources to reduce the costs of treatment for the melioidosis patients. ${ }^{22}$

It is noteworthy that this research was the first research in Malaysia, which highlighted the direct medical cost of melioidosis and made a comparison of the price between the duration of stay and patients' status. However, the primary limitation of this study could be seen from the calculation of the direct medical cost for patients with melioidosis, which was based on the information of resources extracted from the medical records. This situation may lead to the underestimation of the direct medical cost. All the costs were based on the medical records instead of the prospectively collected clinical data. The calculation of the cost was made from the first day until the last day of hospital admission.

The parameters collected from the medical records consisted of the treatment, investigation, and procedures received by the patients, the date of the patient's admission, discharge from hospital, and death, and the methods of the diagnosis. In the case of costs, other costs, including direct non-medical costs (transportation) and indirect medical cost were not included in the study due to the limited 
availability of the data. Moreover, this research placed a sole focus on one hospital, and the direct medical cost only represented the minimum estimation of cost from the overall costs for the treatment of melioidosis patients. It is suggested that further analysis of the economic burden of melioidosis patients is performed with proper management of the variables collected. In order to identify the economic burden of melioidosis patient, the direct medical cost (costs related to buying the medical service), the direct non-medical cost (eg, travel cost, accommodation, meals), and indirect cost (eg, productivity loss) should be considered in the data collection. It is also recommended that the total treatment costs in future studies are stratified based on comorbidities in patients to achieve a correct estimation of the treatment cost for melioidosis.

\section{Conclusion}

The management of melioidosis in terms of diagnosis and appropriate treatment is vital to reduce disease severity and mortality. This study has identified the direct medical cost for managing melioidosis inpatients in Hospital Sultanah Bahiyah, Kedah, Malaysia. Further details on the economic costs of melioidosis should be presented in future studies to achieve improved comprehension regarding the disease. By providing the direct medical cost of melioidosis patients, the study underscores the importance of preventing melioidosis and promote the essential to make useful guidelines in controlling the disease in terms of resources.

\section{Acknowledgments}

The project was supported by the internal grant of Universiti Sultan Zainal Abidin (grant number: UniSZA/ 2018/DPU/16).

\section{Disclosure}

The authors report that no conflicts of interest are involved in this research.

\section{References}

1. Woods KL, Boutthasavong L, NicFhogartaigh C, et al. Evaluation of a rapid diagnostic test for detection of burkholderia pseudomallei in the Lao People's Democratic Republic. J Clin Microbiol. 2018;56(7). doi:10.1128/JCM.02002-17.

2. Nathan S, Chieng S, Kingsley PV, et al. Melioidosis in Malaysia: incidence, clinical challenges, and advances in understanding pathogenesis. Trop Med Infect Dis. 2018;3(1). doi:10.3390/ tropicalmed3010025.

3. Pande K, Kadir KAA, Asli R, Chong VH. Melioidosis in Brunei Darussalam. Trop Med Infect Dis. 2018;3:1-12.
4. Limmathurotsakul D, Golding N, Dance DAB, et al. Predicted global distribution of Burkholderia pseudomallei and burden of melioidosis. Nat Microbiol. 2016;1(1):1-13. doi:10.1038/nmicrobiol.2015.8

5. Hassan MRA, Pani SP, Peng NP, et al. Incidence, risk factors and clinical epidemiology of melioidosis: a complex socio-ecological emerging infectious disease in the Alor Setar region of Kedah, Malaysia. BMC Infect Dis. 2010;10(1):302. doi:10.1186/1471-2334-10-302

6. Kingsley PV, Leader M, Nagodawithana NS, Tipre M, Sathiakumar N, Nascimento AL. Melioidosis in Malaysia: a review of case reports. PLoS Negl Trop Dis. 2016;10(12):1-18. doi:10.1371/ journal.pntd.0005182

7. Peruski LF, Baggett HC, Bhengsri S, et al. Incidence of bacteremic melioidosis in eastern and northeastern Thailand.Am J Trop Med Hyg. 2011;85(1):117-120. doi:10.4269/ajtmh.2011.11-0070

8. Chierakul W, Tisayaticom K, Thamthitiwat S, et al. Economic burden of bacteremic melioidosis in eastern and northeastern, Thailand.Am J Trop Med Hyg. 2013;89(2):369-373. doi:10.4269/ajtmh.13-0148

9. Health Cluster Yemen. Annual report 2017. NTT Docomo. 2017;21(430).

10. Roslani ADRM, Tay ST, Puthucheary SD, Rukumani DV, Sam IC. Short report: predictors of severe disease in melioidosis patients in Kuala Lumpur, Malaysia. Am J Trop Med Hyg. 2014;91 (6):1176-1178. doi:10.4269/ajtmh.14-0354

11. Chan KPW, Low JGH, Raghuram J, Fook-Chong SMC, Kurup A. Clinical characteristics and outcome of severe melioidosis requiring intensive care. Chest. 2005;128(5):3674-3678. doi:10.1016/S00123692(15)52948-6

12. Freitas A, Silva-Costa T, Lopes F, et al. Factors influencing hospital high length of stay outliers. BMC Health Serv Res. 2012;12(1). doi:10.1186/1472-6963-12-265.

13. PMG Secretariat. Guidelines for clinical and public health management of melioidosis in Pahang. Available from: https://www.moh. gov.my/moh/resources/autodownloadimages/589d71f54e729.pdf. Accessed February 26, 2021.

14. Onofri A, Carbonell EA, Piepho HP, Mortimer AM, Cousens RD. Current statistical issues in Weed Res. Weed Res. 2010;50(1):5-24. doi:10.1111/j.1365-3180.2009.00758.x

15. Zueter AR, Yean CY, Abumarzouq M, et al. The epidemiology and clinical spectrum of melioidosis in a teaching hospital in a North-Eastern state of Malaysia: a fifteen-year review. BMC Infect Dis. 2016;16(1):1-11. doi:10.1186/s12879-016-1583-2

16. Suputtamongkol Y, Chaowagul W, Chetchotisakd P, et al. Risk factors for melioidosis and bacteremic melioidosis. Clin Infect Dis. 1999;29(2):408-413. doi:10.1086/520223

17. Sam IC, Puthucheary SD. Melioidosis in children from Kuala Lumpur, Malaysia. Ann Trop Paediatr. 2006;26(3):219-224. doi:10. 1179/146532806X120318

18. Chandrakar S, Dias M. Clinico-epidemiological spectrum of melioidosis: a 2-year prospective study in the western coastal region of India. S Afr J Infect Dis. 2016;31:14-19.

19. Hantrakun V, Kongyu S, Klaytong P, et al. Clinical epidemiology of 7126 melioidosis patients in Thailand and the implications for a national notifiable diseases surveillance system. Open Forum Infect Dis. 2019;6(12). doi:10.1093/ofid/ofz498.

20. Hantrakun V, Chierakul W, Chetchotisakd P, et al. Cost-effectiveness analysis of parenteral antimicrobials for acute melioidosis in Thailand. Trans $R$ Soc Trop Med Hyg. 2015;109(6):416-418. doi: $10.1093 /$ trstmh/trv002

21. Dance DAB, Sihalath S, Rith K, et al. The cost-effectiveness of the use of selective media for the diagnosis of melioidosis in different settings. PLoS Negl Trop Dis. 2019;13(7):1-10. doi:10.1371/journal. pntd.0007598

22. Huyen DTT, Binh NT, Tuan TM, et al. Analyzing trends in hospital-cost payments of patients using ARIMA and GIS: case study at the Hanoi medical university hospital, Vietnam. $J$ Med Imaging Heal Informatics. 2017;7(2):421-429. doi:10.1166/jmihi.20 17.2111 


\section{Publish your work in this journal}

ClinicoEconomics and Outcomes Research is an international, peerreviewed open-access journal focusing on Health Technology Assessment, Pharmacoeconomics and Outcomes Research in the areas of diagnosis, medical devices, and clinical, surgical and pharmacological intervention. The economic impact of health policy and health systems organization also constitute important areas of coverage. The manuscript management system is completely online and includes a very quick and fair peer-review system, which is all easy to use. Visit http://www.dovepress.com/testimonials.php to read real quotes from published authors. 